\title{
ON THE SUBSTITUTION PROBLEM FOR FREE GROUPS
}

PAUL E. SCHUPP

By a substitution problem for a group $G$ we mean the following. Let $F=\left\langle\alpha_{1}, \cdots, \alpha_{n}\right\rangle$ be a free group. Let $G$ be a group with presentation $\left\langle a_{1}, \cdots, a_{m} ; R_{1}, \cdots, R_{l}\right\rangle$. Suppose we are given a reduced word $W\left(\alpha_{1}, \cdots, \alpha_{n}\right)$. We wish to decide, for $x \in G$ (where $x$ is expressed in the generators $a_{1}, \cdots, a_{m}$ of $G$ ), whether or not $x$ is of the form $W$, that is, to decide if there exist $u_{1}, \cdots, u_{n}$ in $G$ such that $x=W\left(u_{1}, \cdots, u_{n}\right)$.

There exist groups in which it is even impossible to decide if an element is a commutator [1]. The question of solving substitution problems for free groups seems to arise in several contexts and we henceforth restrict ourselves to the case where $G$ is free. Even here, the only results we know are that one can decide if an element of a free group is a $k$ th power and the elegant method of Wicks [4] for deciding if an element of a free group is a commutator. This note presents a proof that the substitution problem is solvable for $G$ a free group and $W(\alpha, \beta)$ any element of the free group on $\alpha$ and $\beta$.

Lemma. Let $F=\left\langle\alpha_{1}, \cdots, \alpha_{n}\right\rangle$ and $G=\left\langle a_{1}, \cdots, a_{m}\right\rangle$ be free groups. Let $W \in F$, and let $x \in G$. Suppose that $W\left(u_{1}, \cdots, u_{n}\right)=x$ for some $u_{1}, \cdots, u_{n}$ in $G$. Then there exist $W^{*} \in F$ and $v_{1}, \cdots, v_{n}$ in $G$ such that:

(i) The set $\left\{v_{1}, \cdots, v_{n}\right\}$ is Nielsen reduced.

(ii) $W^{*}\left(v_{1}, \cdots, v_{n}\right)=x$ and $W^{*}$ is the image of $W$ under an automorphism of $F$.

Proof. We regard Nielsen transformations as acting on $n$-tuples (cf. [2]). Now there is a sequence $T_{1}, \cdots, T_{k}$ of Nielsen transformations and an $n$-tuple $\left(v_{1}, \cdots, v_{n}\right)$ of Nielsen reduced elements such that $\left(u_{1}, \cdots, u_{n}\right) \rightarrow\left(v_{1}, \cdots, v_{n}\right)$ by successively applying the transformations $T_{i}$. We use the same letters to denote both the corresponding Nielsen transformations applied to $n$-tuples of generators of $F$ and the automorphisms of $F$ which the transformations induce. Thus if $T_{1}$ is the Nielsen transformation sending $\left(a_{1} a_{2}^{-1}, a_{2}\right) \rightarrow\left(a_{1}, a_{2}\right)$, then $\left(\alpha_{1}, \alpha_{2}\right) \rightarrow\left(\alpha_{1} \alpha_{2}^{-1}, \alpha_{2}\right)$ under $T_{1}^{-1}$ and the word $\alpha_{1} \alpha_{2}^{2} \alpha_{1} \rightarrow \alpha_{1} \alpha_{2} \alpha_{1} \alpha_{2}^{-1}$ under the automorphism $T_{1}^{-1}$ induced by the Nielsen transformation. Suppose that $W \rightarrow W^{*}$ by the successive application of the auto-

Received by the editors December 9, 1968. 
morphisms $T_{1}^{-1}, \cdots, T_{k}^{-1}$ of $F$. Then $W^{*}\left(v_{1}, \cdots, v_{n}\right)=x$ and $W^{*}$ is the image of $W$ under an automorphism of $F$.

For $y \in G$, let $|y|$ denote the length of $y$ with respect to the generators $a_{1}, \cdots, a_{m}$. Similarly, for $Z \in F$, let $|Z|$ denote the length of $Z$ with respect to $\alpha_{1}, \cdots, \alpha_{n}$. We will show that one can assume that $\left|v_{i}\right| \leq|x|, i=1, \cdots, n$, and, if for those $\alpha_{i}$ which actually occur in $W^{*}, v_{i} \neq 1$, then $\left|W^{*}\right| \leq|x|$. If $\alpha_{j}$ does not occur in $W^{*}$, then $v_{j}$ can be replaced by 1 without altering the fact that the $v_{i}$ are Nielsen reduced and that $W^{*}\left(v_{1}, \cdots, v_{n}\right)=x$.

Let $t_{q}$ denote one of the $\left(v_{i}\right)^{ \pm 1}$. Two cancellation properties of a Nielsen reduced set of nontrivial elements are that if $t_{1} \cdots t_{p}$ is a product with no $t_{q}=t_{q+1}^{-1}$ then

(i) $\left|t_{1} \cdots t_{p}\right| \geq p$, and

(ii) $\left|t_{1} \cdots t_{p}\right| \geq \max _{1 \leq q \leq p}\left|t_{q}\right|$.

Since $W^{*}\left(v_{1}, \cdots, v_{n}\right)=x$ and we are assuming that no $v_{i}$ which appears is 1 , we conclude from (i) that $\left|W^{*}\right| \leq|x|$. By (ii), if $\alpha_{j}$ occurs in $W^{*}$, then $\left|v_{j}\right| \leq|x|$.

Now by Whitehead's Theorem on the automorphisms of a free group [3], we can effectively list all words $W^{\prime} \in F$ such that $\left|W^{\prime}\right|$ $\leq|x|$ and $W^{\prime}$ is the image of $W$ under an automorphism of $F$. We can then enumerate all $n$-tuples $\left(u_{1}^{\prime}, \cdots, u_{n}^{\prime}\right)$ such that maximum $\left|u_{i}^{\prime}\right|$ does not exceed $|x|$ and substitute all such $n$-tuples into the $W^{\prime}$ obtained above. Since $W^{\prime}$ is obtained from $W$ by an automorphism, $W^{\prime}\left(u_{1}^{\prime}, \cdots, u_{n}^{\prime}\right)=x$ if and only if there exist $u_{1}, \cdots, u_{n}$ such that $W\left(u_{1}, \cdots, u_{n}\right)=x$.

So far, all that we have done is independent of the number of $\alpha_{i}$. The difficulty is that we have, in general, no algorithm to decide if a word on the $\alpha$ 's can be obtained from $W$ by an automorphism and then replacing some letters by 1 . However, the situation is manageable if $n=2$.

TheOREM. Let $F=\langle\alpha, \beta\rangle$. Let $W$ be a reduced word of $F$. Let $G$ be a free group. Then there is an algorithm to decide, for $x \in G$, if $x$ is of the form $W$.

Proof. Let $\sigma_{\alpha}(Y)$ denote the exponent sum of $\alpha$ in the word $Y$. Let $a=\sigma_{\alpha}(W)$, let $b=\sigma_{\beta}(W)$, and let $d$ be the greatest common divisor of $a$ and $b$. Since there exist integers $s, t$, such that $d=a s+b t$, we have $v^{d}=W\left(v^{s}, v^{t}\right)$. Thus if $x \in G$ is a $d$ th power, then $x$ is of the form $W$. Suppose $x$ is not a $d$ th power and $W\left(u_{1}, u_{2}\right)=x$. Then in the process of obtaining a Nielsen reduced pair $\left(v_{1}, v_{2}\right)$, neither $v_{1}$ nor $v_{2}$ is 1 . For if we had, say $v_{1}=1$, then $x=v_{2}^{7}$ where $r$ is a multiple of $d$. Hence $x$ is either a $d$ th power or we can decide if $x$ is of the form $W$ by examining a finite number of substitutions. 


\section{BIBLIOGRAPHY}

1. G. Baumslag, W. W. Boone and B. H. Neumann, Some unsolvable problems about elements and subgroups of groups, Math. Scand. 7 (1959), 191-201.

2. W. Magnus, A. Karrass and D. Solitar, Combinatorial group theory, Interscience, New York, 1966.

3. J. H. C. Whitehead, On equivalent sets of elements in a free group, Proc. London Math Soc. 41 (1936), 48-56.

4. M. J. Wicks, Commutators in free products, London Math. Soc. 37 (1962), 433444.

UNIVERSITY OF ILLINOIS, URBANA 